\title{
Expressão de caracteres reprodutivos e vegetativos de Senna velutina (Vogel) H. S. Irwin \& Barneby (Leguminosae, Caesalpinioideae) em dois ambientes distintos de cerrado
}

\author{
PATRÍCIA THIEME ONOFRI SAIKI ${ }^{1,2}$, BETHAYNE SILVA $^{1}$ e CECÍLIA LOMÔNACO ${ }^{1}$
}

(recebido: 04 de outubro de 2007; aceito: 08 de maio de 2008)

\begin{abstract}
Expression of reproductive and vegetative characters of Senna velutina (Vogel) H. S. Irwin \& Barneby (Leguminosae, Caesalpinioideae) in two distinct "cerrado" environments). The objective of this work was to verify the influence of two distinct "cerrado" environments in the expression of reproductive and vegetative characters of Senna velutina. Ten individuals were randomly selected in each area in order to characterize plant height, number of flowers and fruits, fruit length, seed number and biomass, flowering period, foliole shape, stomata density and dimensions. Population density and spatial distribution were also verified. Fruit number and length, seed number and biomass were significant larger in the "cerrado" area when compared to palm swamp border area. On the other hand, the number of flowers and buds was significantly larger in the palm-swamp border area. No difference was detected between areas on plant height, foliole length and width, foliolar index, inflorescence length, number of viable seeds, stomata length and width and stomata density. The flowering period started lately in the palm swamp border area, but the reproductive period in the "cerrado" area was longer than the in palm swamp border area. A contagious spatial distribution was verified in both subpopulations. The phenotypic variations registered in S. velutina were restricted to reproductive traits, indicating large canalization and herdability on its somatic characteristics. The "cerrado" area offered better conditions for S. velutina reproductive success comparing to the palm swamp border area.
\end{abstract}

Key words - ecotypes, environmental heterogeneity, phenotypic variation

RESUMO - (Expressão de caracteres reprodutivos e vegetativos de Senna velutina (Vogel) H. S. Irwin \& Barneby (Leguminosae, Caesalpinioideae) em dois ambientes distintos de cerrado). Este trabalho verificou a influência de dois ambientes distintos de cerrado na expressão de caracteres reprodutivos e vegetativos de Senna velutina. Casualmente, dez indivíduos de cada área foram escolhidos para caracterização de altura, número de flores, frutos e sementes, comprimento dos frutos, biomassa de sementes, fenofases de floração, formato foliolar, densidade e dimensões estomáticas. A densidade de ocorrência da espécie e o tipo de distribuição espacial foram também verificados. Na área de cerrado, o número e comprimento de frutos, o número e biomassa das sementes foram maiores quando comparados aos valores obtidos na borda de vereda. Por outro lado, o número de flores e botões foi maior no ambiente de borda de vereda. Não foram detectadas diferenças entre as áreas quanto à altura dos indivíduos, comprimento e largura dos folíolos, índice foliolar, comprimento das inflorescências, número de sementes viáveis, comprimento e largura dos estômatos e densidade estomática. O período de floração na vereda iniciou-se mais tardiamente, mas o período reprodutivo observado no cerrado sensu stricto foi mais longo do que na borda de vereda. Ambas subpopulações apresentaram padrão agregado de distribuição espacial. As respostas fenotípicas às diferenças ambientais registradas restringiram-se às estruturas reprodutivas, indicando haver maior canalização e, portanto, maior herdabilidade nas estruturas somáticas. O cerrado ofereceu condições mais favoráveis ao sucesso reprodutivo de S. velutina do que a borda de vereda.

Palavras-chave - ecótipos, heterogeneidade ambiental, variabilidade fenotípica

\section{Introdução}

Uma forma de adaptação dos organismos à heterogeneidade ambiental é a sua especialização local, a partir da expressão de caracteres com baixa

\footnotetext{
1. Universidade Federal de Uberlândia, Instituto de Biologia, Caixa Postal 593, 384000-902 Uberlândia - MG, Brasil.

2._Autor para correspondência: patriciathieme@yahoo.com.br
}

herdabilidade, de acordo com as demandas ambientais (Scheiner \& Callahan 1999). A capacidade de um organismo responder aos fatores ambientais, alterando seu fenótipo sem a necessidade de que essas modificações sejam genéticas, é denominada plasticidade fenotípica (Bradshaw 1965, Vlot \& Bachmann 1991).

A plasticidade fenotípica pode ainda estar relacionada a processos de especialização e formação de ecótipos por seleção disruptiva sendo, portanto, um importante 
mecanismo gerador de variabilidade (Thompson 1991, Schmid 1992, Coleman et al. 1994, Via et al. 1995, Mal \& Lovett-Doust 2005).

O Cerrado brasileiro, sendo uma formação vegetal complexa pode, naturalmente, favorecer a expressão da plasticidade fenotípica em resposta à heterogeneidade das condições ambientais. Este domínio fitogeográfico abriga uma grande variedade de fisionomias e é caracterizado por apresentar grande variação edáfica quanto à textura, fertilidade e composição mineral (Goodland \& Pollard 1973).

O estudo da plasticidade fenotípica pode ter grande importância no manejo de ecossistemas, na compreensão da estruturação de comunidades vegetais, na elucidação de teorias evolutivas que buscam explicar a origem da variabilidade dos organismos, como também pode ser utilizada como ferramenta nos processos de melhoramento de plantas (Walker \& Noy-Meir 1982, Donohue 2003).

Neste contexto, este trabalho teve como objetivo verificar a influência de dois ambientes distintos de Cerrado na expressão de caracteres reprodutivos e vegetativos de Senna velutina (Vogel) H. S. Irwin \& Barneby (Leguminosae, Caesalpinioideae).

\section{Material e métodos}

Área de estudo - O estudo foi desenvolvido na Reserva Vegetal do Clube Caça \& Pesca Itororó de Uberlândia, MG $\left(18^{\circ} 55^{\prime} \mathrm{S}\right.$ e $\left.48^{\circ} 17^{\prime} \mathrm{O}\right)$, que possui 127 ha, preservando áreas de cerrado sensu stricto, campo sujo e vereda. Esta se estabelece em áreas de nascentes, cuja vegetação é predominantemente herbácea (Appolinário \& Schiavini 2002), apresentando alguns arbustos largamente espaçados. A área de cerrado, por sua vez, apresenta menor cobertura herbácea, menor espaçamento entre arbustos e presença de algumas árvores. Como a transição entre as áreas é gradual, com delimitação imperceptível visualmente, a coleta de dados foi feita desconsiderando a zona de transição.

O clima da região apresenta duas estações bem definidas, uma seca e outra chuvosa. A temperatura média anual é de $22{ }^{\circ} \mathrm{C}$ e os índices pluviométricos oscilam em torno de $1550 \mathrm{~mm}$ ao ano (Nimer \& Brandão 1989). A coleta de dados ocorreu no período de abril a novembro de 2005 .

Espécie estudada - Senna velutina é uma Leguminosae decídua de porte arbustivo. Suas folhas são compostas e suas inflorescências terminais, com flores pentâmeras e hermafroditas. As pétalas são amarelas e os frutos são do tipo deiscente. A antese das flores é diurna, com duração de 24 horas e, quando polinizadas, apresentam alterações na coloração, iniciando o processo de senescência (S. M. Carvalho, dados não publicados).

Caracteres morfológicos - Foram selecionados aleatoriamente e marcados 10 indivíduos na área de cerrado sensu stricto e
10 indivíduos na borda de vereda, distando, no mínimo, $10 \mathrm{~m}$ entre si. Esses indivíduos foram medidos quanto à altura, utilizando-se uma trena. Em cada indivíduo foram observadas seis inflorescências para caracterização das fenofases de floração: botão verde (com coloração verde), botão maduro (com coloração amarelada), flor em antese (com pétalas e sépalas totalmente abertas), flor senescente (já polinizada ou abortada, apresentando peças florais murchas), fruto jovem (em início de desenvolvimento) e fruto desenvolvido (com mais de $10 \mathrm{~cm}$ de comprimento). As inflorescências foram medidas com fita métrica e nelas foi contado o número de estruturas reprodutivas por fenofase. A observação das inflorescências foi feita em um único dia nas duas áreas e observações complementares acerca da duração do período de floração e frutificação foram realizadas mensalmente, de abril, quando a espécie iniciava seu período reprodutivo, a setembro de 2005.

Em cada área, foram coletados 60 frutos desenvolvidos, seis por indivíduo, medidos quanto ao comprimento, com auxílio de fita métrica. Foram quantificadas as sementes totais e as viáveis de cada fruto, das quais cinco foram aferidas quanto ao comprimento e largura com uso de paquímetro digital para estimar sua biomassa (comprimento x largura). Foram consideradas viáveis as sementes morfologicamente bem formadas e não predadas. Não foram realizados testes de viabilidade do embrião. A taxa de predação de estruturas reprodutivas foi estimada para cada área, calculando-se o número de estruturas predadas em relação ao número total de estruturas observadas.

Dos 10 indivíduos marcados de cada área, foram coletadas quatro folhas, provenientes dos quinto e sextos nós, localizados imediatamente abaixo de uma inflorescência. Nestas folhas foi medido, com o auxílio de paquímetro digital, o segundo par de folíolos, quanto ao comprimento (C) e, no ponto médio, a largura (L). Somente foram considerados folíolos bem formados e não predados. Para caracterização do formato do folíolo foi calculado o índice foliolar (IF), utilizando-se a fórmula $\mathrm{IF}=\mathrm{C} \mathrm{L}^{-1}$. Folhas alongadas apresentam $\mathrm{IF}>1$, folhas largas possuem IF $<1$, enquanto que, em folhas arredondadas (tão largas quanto longas) o IF $=1$.

Para estimar a densidade de estômatos na superfície adaxial (Laboriau et al. 1961) foram coletados dois folíolos medianos de cinco indivíduos, escolhidos ao acaso em cada área. Desses folíolos extraiu-se a impressão foliolar da epiderme a partir da confecção de molde, feito com uso de esmalte incolor. O molde foi elaborado tomando-se a região mediana do folíolo e a contagem do número de estômatos foi feita utilizando-se 20 campos, quatro por indivíduo, em uma área de $1 \mathrm{~mm}^{2}$.

Para obtenção da medida do comprimento e largura de 25 estômatos de cada área, foram feitos cortes paradérmicos à mão livre. Os cortes foram incluídos em solução de ácido nítrico e ácido crômico por nove horas, sendo em seguida lavados com água destilada e corados com safranina aquosa a 1\%, para a confecção de lâminas com uso de água glicerinada (Johansen 1940). As medidas foram feitas em microscópio utilizando ocular graduada em micrômetros. 
Para estimar a densidade populacional foram sorteadas 20 parcelas de $10 \mathrm{~m}^{2} \mathrm{em}$ cada área para contagem do número de indivíduos. A distribuição espacial dos indivíduos nas áreas amostradas foi analisada por meio do Índice de Dispersão (Brower et al. 1997).

Análises estatísticas - Diferenças significativas entre as áreas nas variáveis obtidas foram verificadas pelo teste de MannWhitney. Uma tabela de contingência foi construída para verificar se a freqüência das estruturas reprodutivas por fenofase diferia entre as áreas de coleta. Foi obtida, para cada área, uma matriz de correlação de Pearson para verificar se havia relação entre as variáveis coletadas. Os índices de correlação obtidos nas áreas de coleta foram comparados entre si pelo teste $Z$ (Zar 1984) para verificar se havia divergências na associação entre os caracteres em cada ambiente. A análise comparativa da correlação de caracteres entre ambientes informa não somente o grau de associação entre tais caracteres (i.e. pleiotropia), mas também se influências ambientais e respostas plásticas estão afetando a expressão destas características (Cheverud 1988, Falconer 1989). Os testes estatísticos foram realizados utilizando-se o programa SYSTAT ${ }^{\circledR}$ para Windows ${ }^{\circledR}$ versão 10.2.

\section{Resultados}

Senna velutina apresentou diferenças significativas entre as áreas em caracteres reprodutivos (tabela 1). $\mathrm{Na}$ área de cerrado o número de frutos, o comprimento dos frutos, o número de sementes por fruto e a biomassa das sementes foram maiores quando comparados aos valores obtidos na borda de vereda. Por outro lado, o número de flores e botões foi significativamente maior no ambiente de borda de vereda. A predação das estruturas reprodutivas foi muito reduzida em ambas as áreas $(0,02 \%$ na borda de vereda e 0,04\% na área de cerrado).

Foi também constatada diferença no número de estruturas reprodutivas segundo os estágios de floração e frutificação $\left(\chi^{2}=342,22 ; P<0,001\right)$ (figura 1 ), pois enquanto as plantas do cerrado já apresentavam frutos desenvolvidos, nas plantas da vereda ainda predominavam as flores senescentes. Observações ao longo do período reprodutivo confirmaram a notificação de que a floração e a frutificação iniciaram-se tardiamente na borda de

Tabela 1. Comparações, feitas pelo teste de Mann-Whitney $(U)$ e caracterização fenotípica (mediana e amplitude) de estruturas reprodutivas, vegetativas e da densidade de ocorrência de subpopulações de Senna velutina, nas áreas de cerrado sensu stricto e borda de vereda da Reserva Vegetal do Clube Caça \& Pesca Itororó de Uberlândia, MG (A = amplitude; $n=$ tamanho da amostra). Resultados significativos $(P<0,05)$ estão em negrito.

Table 1. Comparisons, made by Mann-Whitney test $(U)$ and phenotypic characterization (median and range) of reproductive, vegetative traits and density of occurrence of Senna velutina, at "cerrado" and palm-swamp border areas of the "Reserva Vegetal do Clube Caça e Pesca Itororó de Uberlândia", MG (A = range; $n=$ sample size). Significant results $(P<0,05)$ are in boldface.

\begin{tabular}{|c|c|c|c|c|c|c|c|c|}
\hline \multirow{2}{*}{ Caracteres analisados } & \multicolumn{3}{|c|}{ Cerrado } & \multicolumn{3}{|c|}{ Borda de vereda } & \multirow{2}{*}{$U$} & \multirow{2}{*}{$P$} \\
\hline & mediana & A & $n$ & mediana & A & $\mathrm{n}$ & & \\
\hline Altura das plantas (m) & 1,50 & 1,71 & 10 & 1,24 & 1,29 & 10 & 33,00 & 0,199 \\
\hline $\begin{array}{l}\text { Densidade de ocorrência } \\
\text { (ind. } 100 \mathrm{~m}^{-2} \text { ) }\end{array}$ & 1,00 & 7,00 & 20 & 0,00 & 3,00 & 20 & 129,00 & $\mathbf{0 , 0 3 5}$ \\
\hline Índice Foliolar & 2,43 & 1,20 & 68 & 2,45 & 1,61 & 68 & 2324,50 & 0,957 \\
\hline Comprimento dos folíolos (mm) & 75,55 & 52,40 & 68 & 76,77 & 51,58 & 68 & 2503,00 & 0,406 \\
\hline Largura dos folíolos (mm) & 31,29 & 45,34 & 68 & 31,13 & 25,44 & 68 & 2340,50 & 0,901 \\
\hline Comprimento estômatos $(\mu \mathrm{m})$ & 26,00 & 9,00 & 25 & 28,00 & 10,00 & 25 & 264,00 & 0,343 \\
\hline Largura estômatos $(\mu \mathrm{m})$ & 23,00 & 11,00 & 25 & 24,00 & 11,00 & 25 & 288,00 & 0,630 \\
\hline $\begin{array}{l}\text { Densidade estomática } \\
\text { (estômatos } \mathrm{mm}^{-2} \text { ) }\end{array}$ & 61,74 & 49,98 & 20 & 68,60 & 37,68 & 20 & 14,00 & 0,754 \\
\hline $\begin{array}{l}\text { Número de flores e } \\
\text { botões por inflorescência }\end{array}$ & 5,00 & 48,00 & 60 & 28,00 & 90,00 & 60 & 84,00 & $\mathbf{0 , 0 1 0}$ \\
\hline $\begin{array}{l}\text { Comprimento das } \\
\text { inflorescências }(\mathrm{cm})\end{array}$ & 15,20 & 20,80 & 15 & 18,90 & 16,10 & 15 & 145,50 & 0,171 \\
\hline Número de frutos & 36,50 & 40,00 & 60 & 17,50 & 27,00 & 60 & 6,50 & 0,001 \\
\hline Comprimento dos frutos $(\mathrm{cm})$ & 20,45 & 21,50 & 60 & 17,80 & 11,00 & 60 & 1074,50 & $<0,001$ \\
\hline Número de sementes por fruto & 34,00 & 31,00 & 60 & 32,50 & 25,00 & 60 & 1423,00 & 0,048 \\
\hline Número de sementes viáveis por fruto & 26,50 & 38,00 & 60 & 26,00 & 32,00 & 60 & 1819,00 & 0,920 \\
\hline Biomassa das sementes $\left(\mathrm{mm}^{2}\right)$ & 13,91 & 19,26 & 289 & 11,51 & 16,75 & 289 & 28460,50 & $<0,001$ \\
\hline
\end{tabular}




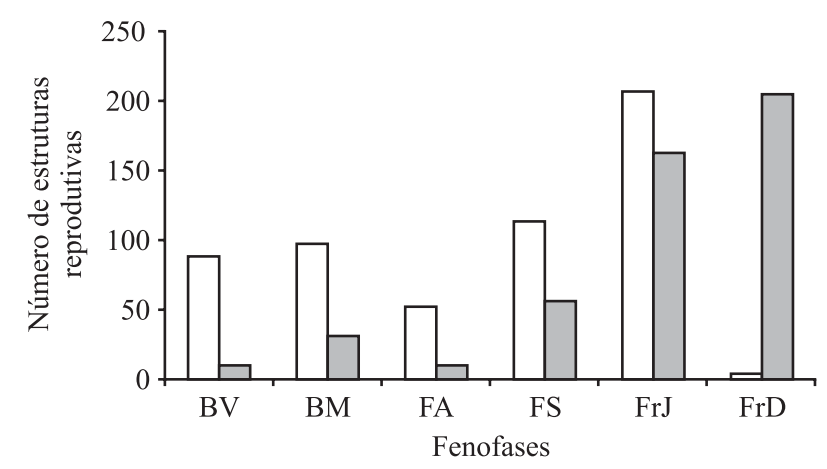

Figura 1. Número de estruturas reprodutivas por estádio de floração em indivíduos de Senna velutina da Reserva Vegetal do Clube Caça \& Pesca Itororó de Uberlândia, MG, em áreas de cerrado senso stricto e borda de vereda $(\mathrm{BV}=$ botões verdes; $\mathrm{BM}=$ botões maduros; $\mathrm{FA}=$ flores em antese; $\mathrm{FS}=$ flores senescentes; FrJ = frutos jovens; $\mathrm{FrD}=$ frutos desenvolvidos; $\square=$ borda de vereda; $\square=$ cerrado).

Figure 1. Number of reproductive traits per flowering stage in Senna velutina individuals of the "Reserva Vegetal do Clube Caça e Pesca Itororó de Uberlândia", MG, in the "cerrado" and palm-swamp border areas (BV = young buds; $\mathrm{BM}=$ mature buds; $\mathrm{FA}=$ flowers in anthesis; $\mathrm{FS}=$ senescence flowers; $\mathrm{FrJ}=$ young fruits; $\mathrm{FrD}=$ developed fruits; $\square=$ palmswamp border; $\square=$ "cerrado").

vereda. Além disto, o período reprodutivo no ambiente de cerrado foi mais longo (de abril a setembro) do que na borda de vereda. Ao final da estação seca (setembro), os indivíduos do cerrado ainda possuíam alguns frutos maduros, enquanto na borda de vereda as plantas não mais apresentam estruturas reprodutivas.
Não foram detectadas diferenças significativas entre as áreas quanto a altura dos indivíduos, comprimento dos folíolos, largura dos folíolos, índice foliolar, comprimento das inflorescências, número de sementes viáveis, comprimento dos estômatos, largura dos estômatos e densidade estomática (tabela 1). Os folíolos foram caracterizados como largos (IF > 1) e não diferiram entre as áreas ( $\mathrm{IF}=2,45 \pm 0,23$ no cerrado $\mathrm{e}$ $\mathrm{IF}=2,47 \pm 0,34$ na borda de vereda).

A densidade populacional dessa espécie diferiu entre as áreas de coleta, sendo maior no cerrado (tabela 1). Os Índices de Dispersão para os ambientes de borda de vereda e cerrado foram, respectivamente, $\mathrm{ID}_{\mathrm{bv}}=1,62 \mathrm{e}$ $\mathrm{ID}_{\mathrm{c}}=3,32$, indicando, para ambos, distribuição agregada.

Os índices de correlação obtidos não diferiram significativamente entre as áreas. As relações entre as variáveis foram todas significativas, com exceção das correlações entre o comprimento dos frutos e o número de sementes viáveis com a biomassa das sementes na área de borda de vereda e entre altura dos indivíduos e número de estruturas reprodutivas em ambas as áreas (tabela 2).

\section{Discussão}

Nota-se a influência do ambiente na expressão de alguns caracteres morfológicos de Senna velutina, especialmente aqueles ligados à reprodução. A área de cerrado parece oferecer condições mais favoráveis à sobrevivência e perpetuação da espécie, o que pode ser evidenciado pela maior densidade de indivíduos nesta área e, ainda, pela maior produtividade, refletida na

Tabela 2. Comparação, feita pelo teste $Z$, dos índices de correlação de Pearson $(r)$ entre caracteres fenotípicos de indivíduos de Senna velutina, nas áreas de cerrado sensu stricto dito e borda de vereda da Reserva Vegetal do Clube Caça \& Pesca Itororó de Uberlândia, MG.

Table 2. Comparisons made by $Z$ test of the Pearson correlation index ( $r$ ) between phenotypic traits of Senna velutina individuals in the "cerrado" and palm-swamp border areas of the "Reserva Vegetal do Clube Caça e Pesca Itororó de Uberlândia", MG.

\begin{tabular}{|c|c|c|c|c|c|c|}
\hline \multirow{2}{*}{ Caracteres analisados } & \multicolumn{2}{|c|}{ Cerrado } & \multicolumn{2}{|c|}{ Borda de vereda } & \multirow{2}{*}{$Z$} & \multirow{2}{*}{$P$} \\
\hline & $r$ & $P$ & $r$ & $P$ & & \\
\hline comprimento fruto $\mathrm{x} \mathrm{n}^{\mathrm{o}}$ sementes & 0,593 & $<0,001$ & 0,659 & $<0,001$ & 0,737 & $>0,05$ \\
\hline comprimento fruto $\mathrm{x}$ biomassa sementes & 0,469 & $<0,001$ & 0,115 & 0,383 & 0,312 & $>0,05$ \\
\hline comprimento fruto $\times n^{\circ}$ sementes viáveis & 0,287 & 0,026 & 0,576 & $<0,001$ & 0,476 & $>0,05$ \\
\hline $\mathrm{n}^{\mathrm{o}}$ sementes $\mathrm{x}$ biomassa sementes & 0,288 & 0,026 & $-0,269$ & 0,038 & 0,286 & $>0,05$ \\
\hline $\mathrm{n}^{\mathrm{o}}$ sementes $\mathrm{x} \mathrm{n}^{\mathrm{o}}$ sementes viáveis & 0,389 & 0,002 & 0,758 & $<0,001$ & 0,701 & $>0,05$ \\
\hline biomassa sementes $\mathrm{x}$ n⿳o sementes viáveis & $-0,308$ & 0,017 & $-0,196$ & 0,134 & 0,258 & $>0,05$ \\
\hline comprimento inflorescência $\times \mathrm{n}^{\circ}$ flores & 0,900 & $<0,001$ & 0,621 & 0,014 & 1,099 & $>0,05$ \\
\hline altura $\mathrm{x} \mathrm{n}^{\circ}$ estruturas reprodutivas & 0,218 & 0,546 & $-0,032$ & 0,931 & 0,127 & $>0,05$ \\
\hline
\end{tabular}


quantidade e comprimento de frutos, número e biomassa de sementes.

Embora o número total de sementes por fruto tenha sido significativamente diferente entre as áreas, o mesmo não ocorreu com o número de sementes viáveis, o que indica que as plantas da borda de vereda produzem menos sementes inviáveis do que as plantas do cerrado. A maior produção de sementes viáveis pelas plantas de borda de vereda pode estar associada ao seu maior investimento em estruturas reprodutivas (flores), a fim de maximizar o sucesso da polinização, pois sabe-se que sementes inviáveis podem ser resultantes de óvulos não-fertilizados (Janzen 1978, Bawa \& Buckley 1989). Isto parece indicar que as plantas da borda de vereda poderiam estar compensando o menor número de indivíduos com um aumento na produção de flores, para aumentar sua capacidade reprodutiva. Padrão similar de variação fenotípica em caracteres reprodutivos foi observado em Byrsonima intermedia A. Juss., uma Malpighiaceae estudada neste mesmo ecótono (Rocha-Filho \& Lomônaco 2006), o que permite supor que esta estratégia possa ser comum em plantas que ocorrem em baixa densidade em áreas de Cerrado. O investimento na produção de flores certamente aumenta a atratividade das plantas para seus visitantes florais, que são constituídos principalmente de abelhas que realizam polinização vibrátil ou "buzz pollination" (Gottsberger \& Silberbauer-Gottsberger 1988).

Não foram detectados mecanismos de compensação energética ou "trade-offs" (Stearns 1989) entre o número e a biomassa das sementes, uma vez que ambos foram maiores no ambiente de cerrado. Esses dados contrapõem os da meliácea Cabralea canjerana subsp. polytricha (A. Juss) T. D. Penn. (Fuzeto \& Lomônaco 2000) e da mirtácea Eugenia calycina Cambess. (Cardoso \& Lomônaco 2003), que produziram sementes maiores e em menor número na vereda. A não diferenciação entre as áreas das relações entre caracteres reforça a idéia de que não esteja ocorrendo seleção disruptiva e que, portanto, não são esperadas divergências acentuadas entre as subpopulações com o tempo (Falconer, 1989).

Entretanto, segundo Allaby (1994), ecótipos (populações localmente adaptadas de uma espécie amplamente distribuída) podem ser caracterizados por pequenas variações relacionadas a um hábitat específico. Neste contexto, pode-se afirmar que a heterogeneidade ambiental da área estudada foi suficientemente intensa para produzir respostas plásticas, facilmente detectadas fenotipicamente.

A heterogeneidade ambiental ao longo do ecótono também promoveu diferenciações na duração e no começo do período de floração entre os dois ambientes estudados. Isto poderia ser explicado por diferenças na quantidade de nutrientes edáficos (Sanda \& Amasino 1996, Pigliucci 1997) ou por variações no nível de umidade do solo (Cardoso \& Lomônaco 2003). Diferentes concentrações de nitrogênio, fósforo e potássio no solo já foram relacionadas a respostas fenotípicas quanto ao número de folhas e frutos, altura das plantas e variações no período de floração (Pigliucci et al. 1995, Pigliucci \& Schlichting 1996). Além disso, maior quantidade de fósforo pode estar relacionada com o aumento da biomassa de sementes (Austin 1966, Aarssen \& Burton 1990, Sills $\&$ Nienhuis 1995).

Contrariamente ao exposto para caracteres reprodutivos, não houve interferência do ambiente na expressão de caracteres vegetativos de S. velutina. A não diferenciação da densidade estomática, tamanho dos estômatos, formato e tamanho foliolares e na altura das plantas pode estar refletindo o fato de que as diferenças na umidade relativa e luminosidade entre as áreas não tenham sido grandes o suficiente para induzir respostas plásticas. Sabe-se, que plantas expostas à intensa luminosidade tendem a apresentar folhas menores (Niinemets 1999, Pons et al. 2001, Hoffmann \& Franco 2003) e em menor número (Franco 2004) e que o número e o tamanho de estômatos podem estar relacionados à umidade relativa (Lleras 1977, Fahn \& Cutler 1992).

O padrão agregado de distribuição dos indivíduos deve-se, provavelmente, à dinâmica de dispersão da espécie, cujos frutos espalham as sementes em regiões próximas à planta-mãe. Não se sabe, contudo, se $S$. velutina possui mecanismos assexuados de reprodução, o que também poderia resultar no agrupamento espacial de indivíduos.

As respostas plásticas de S. velutina diferem, em diversos aspectos, das respostas de outras espécies de plantas estudadas na mesma localidade. Por exemplo, Byrsonima intermedia (Malpighiaceae) apresentou diferenças significativas no formato foliar (Rocha-Filho \& Lomônaco 2006) e Cabralea canjerana (Meliaceae) (Fuzeto \& Lomônaco 2000) e Davilla elliptica A. St.-Hil. (Dilleniaceae) (Rocha-Filho \& Lomônaco 2006) formaram plantas mais altas no ambiente do cerrado. Senna velutina também diferiu de E. calycina (Cardoso \& Lomônaco 2003), D. elliptica e B. intermedia na dinâmica de floração, iniciada por estas espécies primeiramente na vereda.

As variações fenotípicas registradas para S. velutina neste estudo restringiram-se às estruturas reprodutivas, o que pode indicar maior canalização e, portanto, maior herdabilidade das estruturas somáticas (Stearns 1989, Weinig 2000). 


\section{Referências bibliográficas}

AARSSEN, L.W. \& BURTON, S.M. 1990. Maternal effects at four levels of Senecio vulgaris (Asteraceae) growth on a soil nutrient gradient. American Journal of Botany 77:1231-1240.

ALLABY, M. 1994. The concise Oxford dictionary of ecology. Oxford University Press, Oxford.

APPOLINÁRIO, V. \& SCHIAVINI, I. 2002. Levantamento fitossociológico de espécies arbóreas de Cerrado (stricto sensu) em Uberlândia - Minas Gerais. Boletim do Herbário Ezechias Paulo Heringer 10:57-75.

AUSTIN, R.B. 1966. The growth of watercress Rorippa nausturtium-aquaticum (L.) Hayeck from seed affected by the phosphorous nutrition of the parent plant. Plant and Soil 24:113-120.

BAWA, K.S. \& BUCKLEY, D.P. 1989. Seed: ovule ratio, selective seed abortion, and mating systems in Leguminosae. In Advances in Legume Biology (C.H. Stirton \& J.L. Zarucchi eds.). Monographs in Systematic Botany from the Missouri Botanical Garden, St-Louis, p.243-262.

BRADSHAW, A.D. 1965. Evolutionary significance of phenotypic plasticity in plants. In Advances in Genetics (E.M. Caspary \& J.M. Thoday, eds.). Academic Press, New York. p.115-155.

BROWER, J.E., ZAR, J.H. \& VON-ENDE, C.N. 1997. Field and laboratory methods of general ecology. McGrawHill, Cheers.

CARDOSO, G.L. \& LOMÔNACO, C. 2003. Variações fenotípicas e potencial plástico de Eugenia calycina Cambess. (Myrtaceae) em uma área de transição cerradovereda. Revista Brasileira de Botânica 26:131-140.

CHEVERUD, J.M. 1988. A comparison of genetic and phenotipic correlations. Evolution 42:958-968.

COLEMAN, J.S., MCCONNAUGHAY, K.D.M. \& ACKERLY, D.D. 1994. Interpreting phenotypic variation in plants. Tree 95:187-191.

DONOHUE, K. 2003. Setting the stage: phenotypic plasticity as habitat selection. International Journal of Plant Science 164:9-92.

FAHN, A. \& CUTLER, D. 1992. Xerophytes. Gebrüder Borntraeger, Berlin.

FALCONER, D.S. 1989. Introduction to quantitative genetics. Hongman Scientific \& Technical, New York.

FRANCO, A.C. 2004. Estratégias funcionais de plantas lenhosas das savanas do Brasil Central: relação ao déficit hídrico e ao regime luminoso. In Fisiologia ecológica em plantas: mecanismos y respuestas a estrés em los ecosistemas (H.M. Cabrera, ed.). EUV, Valparaíso.

FUZETO, P.A. \& LOMÔNACO, C. 2000. Potencial plástico de Cabralea canjerana subsp. polytricha (Adr. Juss.) Penn. (Meliaceae) e seu papel na formação de ecótipos em áreas de cerrado e vereda, Uberlândia, MG. Revista Brasileira de Botânica 23:169-176.
GOODLAND, R.J. \& POLLARD, R. 1973. The Brazilian cerrado vegetation: a fertility gradient. Journal of Ecology 61:219-224.

GOTTSBERGER, G. \& SILBERBAUER-GOTTSBERGER, I. 1988. Evolution of flowers structures and pollination in Neotropical Cassinae (Caesalpiniaceae) species. Phyton 28:293-320.

HOFFMANN, W.A. \& FRANCO, A.C. 2003. Comparative growth analysis of tropical forest and savanna woody plants using phylogenetically independent contrasts. Journal of Ecology 91:475-484.

JANZEN, D.H. 1978. Seedling patterns of tropical trees. In Tropical trees as living systems (P.B. Tomlinson \& M.H. Zimmermann eds.). Cambridge University Press, Cambridge. p.83-128.

JOHANSEN, D.A. 1940. Plant microtechnique. MacGrawHill Book Company, Inc., New York.

LABORIAU, L.G, OLIVEIRA, J.C. \& LABORIAU, F.M.L.S. 1961. Transpiração de Schizolobium parahyba (Vell.) Toledo. Anais da Academia Brasileira de Ciências 33: 348-251.

LLERAS E. 1977. Differences in stomatal number per unit area within the same species under different microenvironmental conditions: a working hypothesis. Acta Amazonica 7:473-476.

MAL, T.K. \& LOVETT-DOUST, J. 2005. Phenotypic plasticity in vegetative and reproductive traits in an invasive weed, Lythrum salicaria (Lythraceae), in response to soil moisture. American Journal of Botany 92:819-825.

NIINEMETS, U. 1999. Components of leaf dry mass per area - thickness and density - alter leaf photosynthetic capacity in reverse directions in wood plants. New Phytologist 144:35-47.

NIMER, E. \& BRANDÃO, A.M.P. 1989. Balanço hídrico e clima da região dos cerrados. Fundação IBGE, Rio de Janeiro.

PIGLIUCCI, M., WHITTON, J. \& SCHLICHTING, D.C. 1995. Reaction norms of Arabidopsis I. Plasticity of characters and correlations across water, nutrient and light gradients. Journal of Evolutionary Biology 8:421-438.

PIGLIUCCI, M. \& SCHLICHTING, D.C. 1996. Reaction norms of Arabidopsis IV. Relationships between plasticity and fitness. Heredity 76:427-436.

PIGLIUCCI, M. 1997. Ontogenetic phenotypic plasticity during the reproductive phase in Arabidopsis thaliana (Brassicaceae). American Journal of Botany 84:887-895.

PONS, T.L., JORDI, W. \& KUIPER, D. 2001. Acclimation of plants to light gradients in leaf canopies: evidence for a possible role for cytokinins transported in the transpiration stream. Journal of Experimental Botany 52:1563-1574.

ROCHA-FILHO, L.C. \& LOMÔNACO, C. 2006. Variações fenotípicas em subpopulações de Davilla elliptica A. St.-Hil. (Dilleniaceae) e Byrsonima intermedia A. Juss. (Malpighiaceae) em uma área de transição cerradovereda. Acta Botanica Brasilica 20:719-725. 
SANDA, S.L. \& AMASINO, R.M. 1996. Ecotype-specific expression of flowering mutant phenotype in Arabidopsis thaliana. Plant Physiology 111:641-644.

SCHEINER, S.M. \& CALLAHAN, H.S. 1999. Measuring natural selection on phenotypic plasticity. Evolution 53:1704-1713.

SCHMID, B. 1992. Phenotypic variation in plants. Evolution Trends in Plants 6:45-59.

SILLS, G.R. \& NIENHUIS, J. 1995. Maternal phenotypic effects due to soil nutrient levels and sink removal in Arabidopsis thaliana (Brassicaceae). American Journal of Botany 82:491-495.

STEARNS, S.C. 1989. The evolutionary significance of phenotypic plasticity. Bioscience 39:436-445.

THOMPSON, J.D. 1991. Phenotypic plasticity as a component of evolutionary change. Trends in Ecology \& Evolution 6:246-249.
VIA, S., GOMULKIEWICZ, R., DEJONG, G., SCHEINER, S.M., SCHLICHTING, C.D. \& VAN TIENDEREN, P.H. 1995. Adaptive phenotypic plasticity: consensus and controversy. Trends in Ecology \& Evolution 19:212-217.

VLOT, E.C. \& BACHMANN, K. 1991. Genetic and nongenetic variation in the number of pappus parts in Microseris douglasii strain D37 (Asteraceae, Lactuceae). Annals of Botany 68:235-241.

WALKER, B.H. \& NOY-MEIR, I. 1982. Aspects of the stability and resilience of savanna ecosystems. In Ecology of tropical savannas: Ecological Studies (B.J. Huntley \& B.H. Walker eds.). Springer, Berlin. p.556-590.

WEINIG, C. 2000. Plasticity versus canalization: population differences in the timing of shade-avoidance responses. Evolution 54:441-451.

ZAR, J.H. 1984. Biostatistical analysis. Prentice Hall, Inc., New Jersey. 